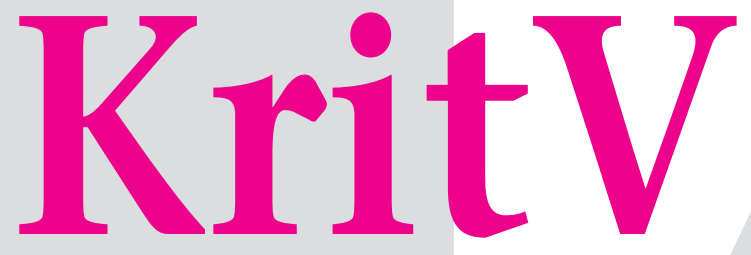

\title{
Kritische Vierteljahresschrift für Gesetzgebung und Rechtswissenschaft
}

\section{Herausgeber}

Peter-Alexis Albrecht

Erhard Denninger

Dieter Grimm

Winfried Hassemer

Christine Hohmann-

Dennhardt

Friedrich Kübler

Jutta Limbach

Ernst Gottfried Mahrenholz

Hans Meyer

Wolfgang Naucke

Lerke Osterloh

Eike Schmidt

Spiros Simitis

Michael Stolleis

Gunther Teubner

Manfred Weiss

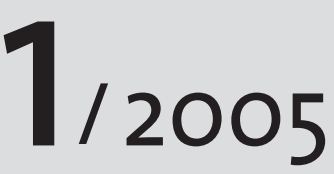

Jahrgang $88 \cdot$ Seiten 1-96 ISSN $0179-2830 \cdot 12666$

\section{Aus dem Inhalt}

Europas Verfasstheit als politische Einheit

Stefan Haack

Deutsche Verfassungsgeschichtsschreibung im 20. Jahrhundert im Spannungsfeld von Politik und Wissenschaft

Ewald Grothe

Brasilien auf der Suche nach einer modernen Verfassung: die Verfassung der Republik der Vereinigten Staaten von Brasilien als kopiertes Original

Wolf Paul

Sanktionen wegen Diskriminierung

Christian Armbrüster

\section{Schulfrieden}

Thorsten Anger

Doppelfunktionelle Nötigungsabsicht bei der Aussageerpressung Jochen Bung

„War on drugs" und Verfassung Plädoyer für die Abschaffung des Brechmitteleinsatzes im Strafverfahren Tobias Mushoff 
Inhaltsverzeichnis

Die Autoren

Stefan Haack

Europas Verfasstheit als politische Einheit 3

Ewald Grothe

Deutsche Verfassungsgeschichtsschreibung im 20. Jahrhundert im Spannungsfeld von Politik und Wissenschaft

Wolf Paul

Brasilien auf der Suche nach einer modernen Verfassung:

die Verfassung der Republik der Vereinigten Staaten von

Brasilien als kopiertes Original

Christian Armbrüster

Sanktionen wegen Diskriminierung

Thorsten Anger

Was bedeutet und wie wichtig ist der »Schulfrieden«?

Zur Lösung religiöser Konflikte in der Schule

Jochen Bung

Doppelfunktionelle Nötigungsabsicht bei der Aussageerpressung

Tobias Mushoff

»War on drugs« und Verfassung - Plädoyer für die Abschaffung

des Brechmitteleinsatzes im Strafverfahren 


\section{Die Autoren}

Dr. Thorsten Anger, Richter am Landgericht Wuppertal, Eiland 1, 42103 Wuppertal

Prof. Dr. Christian Armbrüster, Lehrstuhl für Bürgerliches Recht, Handels- und Gesellschaftsrecht, Privatversicherungsrecht und Internationales Privatrecht an der Freien Universität Berlin, Boltzmannstr. 1, 14195 Berlin

Dr. Jochen Bung, M.A. Wissenschaftlicher Mitarbeiter am Lehrstuhl für Rechtstheorie, Strafrecht und Strafprozessrecht der Johann Wolfgang Goethe-Universität, Senckenberganlage 31, 60325 Frankfurt am Main

Privatdozent Dr. Ewald Grothe, Bergische Universität Wuppertal, Gaußstr. 20, 42097 Wuppertal

Dr. Stefan Haack, Wissenschaftlicher Mitarbeiter am Lehrstuhl für Staats- und Verwaltungsrecht, Wirtschafts-, Umwelt- und Planungsrecht der Universität Leipzig, Postfach 920, 04009 Leipzig

Tobias Mushoff, c/o Lehrstuhl Prof. Dr. Regina Harzer, Fakultät für Rechtswissenschaft, Universitätsstr. 25, 33615 Bielefeld

Prof. Dr. iur. h.c. Wolf Paul, Feldbergstrasse 96D, 61389 Schmitten

\section{Beilagenhinweis:}

Dieser Ausgabe liegt ein Prospekt der Nomos Verlagsgesellschaft bei.

Wir bitten freundlichst um Beachtung. 